Kommentar

\section{Systemrelevanz der Pflegeberufe in Zeiten von Corona - und darüber hinaus}

Die Corona-Pandemie stellt die Gesellschaft vor unvorhergesehene Herausforderungen: Beschränkungen zur Reduktion der sozialen Kontakte werden angeordnet, um eine Ausbreitung des Virus zumindest zu verlangsamen. Das Gesundheitswesen ist mit einem dramatischen Mangel an Schutzausrüstung konfrontiert. Die Anzahl an (Intensiv-)Betten wird deutlich erhöht, um der zunehmenden Zahl an intensivpflichtigen Patientinnen und Patienten gerecht werden zu können. Parallel zu diesen neuen Herausforderungen wird in der aktuellen Situation der bereits seit längerem bestehende Fachkräftemangel in der Pflege vollends ersichtlich.

Obwohl das Gesundheitswesen von großer beschäftigungspolitischer Relevanz ist, verfügt Deutschland im europäischen Vergleich über die schlechteste Relation zwischen Pflegekräften und Patientinnen und Patienten [1]. Die Diskrepanz zwischen Angebot und Bedarf an Fachkräften in der Pflege hat sich bereits in den vergangenen Jahrzehnten deutlich verstärkt [2]. Diese Entwicklung ist u. a. Resultat der bereits vielfach beschriebenen Belastungen im Pflegeberuf [3] sowie des Missverhältnisses zwischen der Leistung der Pflegekräfte, den Rahmenbedingungen, unter denen sie erbracht wird, und der Entlohnung (auch in Form von Lohngerechtigkeit, ausbildungsadäquater Beschäftigung und Entwicklungsmöglichkeiten). Nach dem Modell der „Gratifikationskrise“ kann ein solches Missverhältnis zwischen individueller Verausgabung einer Person und einer als nicht angemessen empfundenen Entschädigung zur Krankheitsentstehung beitragen [4]. Mit Blick auf die Pflege ist darüber hinaus eine gesellschaftliche Krise zu konstatieren, denn mit ihrer mangelnden Anerkennung wird nicht nur ein Individuum verwundbar, sondern das gesamte Versorgungssystem „erkrankt“ und kommt an die Grenzen seiner Leistungsfähigkeit.
Der Fachkräftemangel in der Pflege ist keineswegs neu, jedoch werden seine Auswirkungen durch die Corona-Pandemie verstärkt sichtbar. Bislang wurde die Bedeutung der Pflegeberufe zumeist unter- und die Leidensfähigkeit der Beschäftigten in der Pflege häufig überschätzt. Pflegeberufe haben sich bereits vielfach im Notstand erprobt. Die personelle Krise in der Pflege ist jedoch inzwischen schon Normalzustand geworden. Durch Corona verändert sich jedoch das Bewusstsein der Bevölkerung, da die Wahrscheinlichkeit steigt, diese Dienstleistung unvermittelt in Anspruch nehmen zu müssen. Dies führt neben einer offiziellen Anerkennung der Pflege als systemrelevanter Beruf auch zu Solidaritätsbekundungen vonseiten der Politik und Öffentlichkeit. Wichtig ist an dieser Stelle, dass es nicht nur bei der rhetorischen Solidarität bleibt, sondern die Gesellschaft sich damit auseinandersetzt, was uns Pflege wert ist und wie lange wir es uns tatsächlich noch leisten können, Tätigkeiten in der Pflege nicht adäquat zu honorieren. Daraus müssen politische Entscheidungen und konkrete Handlungen erfolgen.

Dass die Verantwortung, die Pflege für den Menschen übernimmt, nicht angemessen honoriert wird, zeigt sich u. a. darin, dass Produktionsberufe höher entlohnt werden als Pflegeberufe. In Zeiten von Corona wird nun über Sonderprämien für Beschäftige in versorgungsrelevanten Bereichen diskutiert. Dies ist ein erster und wichtiger Schritt, der jedoch einer Verstetigung in einer dauerhaft höheren Entlohnung der Pflegeberufe bedarf. Während der Corona-Pandemie werden - verständlicherweise - die auftretenden „Symptome“ zumeist mit kurzfristigen Maßnahmen bekämpft, damit das System weiterhin arbeitsfähig bleibt. Dazu zählt auch die vorübergehende Aussetzung der Pflegepersonaluntergrenze, welche die Situation für die Pflegenden jedoch nochmals verschlechtert. Unabhängig davon muss langfristig aber v. a. den Ursachen des Fachkräftemangels entgegengetreten werden, um Pflege zukunftsfähig zu gestalten. Hierzu bedarf es einer Verbesserung der Arbeitsbedingungen (z. B. hinsichtlich Flexibilität und Mitsprache in der Dienstplangestaltung), die zu einer Aufwertung des Pflegeberufes und somit auch zu einer Steigerung der Berufsattraktivität führt. Dies ist dringend erforderlich, denn Pflegeberufe sind (zumal in einer alternden Gesellschaft) in der Tat hoch systemrelevant - auch jenseits der der Corona-Pandemie!

Florian Fischer, Lea Raiber, Claudia Boscher, Maik H.-J. Winter.

Hochschule Ravensburg-Weingarten, Institut für Gerontologische Versorgungs- und Pflegeforschung.

\section{Literatur}

[1] Aiken LH, Sermeus W, van den Heede K et al. Patient safety, satisfaction, and quality of hospital care: cross sectional surveys of nurses and patients in 12 countries in Europe and the United States. BMJ 2012; 344: e1717; DOI: 10.1136/bmj.e1717

[2] Simon M. Beschäftigungsstrukturen in Pflegeberufen: Eine Analyse der Entwicklungstrends im Zeitraum 1999 bis 2009. Pflege \& Gesellschaft 2011; 16: 339-371

[3] Höhmann U, Lautenschläger M, Schwarz L. Belastungen im Pflegeberuf: Bedingungsfaktoren, Folgen und Desiderate. In Jacobs K, Kuhlmey A, Greß S, Klauber J, Schwinger A, Hrsg. Pflegereport 2016: Schwerpunkt: Die Pflegenden im Fokus. Stuttgart: Schattauer Verlag; 2016: 73-89

[4] Siegrist ]. Arbeitswelt und stressbedingte Erkrankungen - Forschungsevidenz und präventive Maßnahmen. München: Elsevier Urban \& Fischer; 2015 